\title{
Service Ecosystems Emergence and Interaction: A Simulation Study
}

\author{
Satoru Fujita \\ Hosei University \\ fujita_s@hosei.ac.jp
}

\author{
Claudia Vaughan \\ University of Hawaii at Manoa \\ cmv815@hawaii.edu
}

\author{
Stephen L. Vargo \\ University of Hawaii at Manoa \\ svargo@hawaii.edu
}

\begin{abstract}
This paper describes the examination of emerging institutions and phase transition of service ecosystems in value cocreation processes under the basic tenets of service-dominant logic. We conducted several computational experiments with an agent-based model, in which we represented the generic actors and their operant resources, and examined their interactive behaviors in agent-based simulations. In the simulations, actors started changing their social properties from self-supporting individuals to reciprocal resource integraters. During the transaction, the actors increasingly specialized into specific roles and clusters of actors with the identical roles emerged - pointing towards processes of institutionalization, and dependent on the conditions of land fertility levels. Several phase transitions were observed in emerging service ecosystems, which were supported by complex structures of exchange and collaboration networks.
\end{abstract}

\section{Introduction}

Service-dominant (S-D) logic has been proposed as a theoretical foundation for understanding economic exchange and value cocreation among actors from a service-for-service perspective. In S-D logic, service represents a transcending concept to goods and services, highlighting that economic exchange is primarily about the activities that actors do for others and want done for themselves [11][14][15][16]. This view is relatively orthogonal to the traditional approach, in which exchange and value creation are understood in terms of goods - tangible firm output embedded with value [15]. Consistent with others [14] we refer to such goods-centered thinking as goods-dominant (GD) logic. S-D logic views all actors as generic [17] that is, it disregards the producer-consumer divide, since all market participants mutually engage in service-providing and resource-integrating activities, central to value cocreation. More generally, S-D logic holds that the assignment of predefined roles to market actors unduly restricts our understanding of the mutually interdependent activities of actors from which roles and other institutional structures emerge. S-D logic seeks to understand markets and, at a higher level, whole economies, as a result of these mutual exchange activities and institutional structures that make up service-ecosystems -- relatively self-contained, selfadjusting systems of resource-integrating actors connected by shared institutional arrangements and mutual value creation through service exchange [19]. Arguably, this simplified view allows a clearer vision and understanding of the systemic nature of value creation, by examining the emergence of macro-level phenomena. Emergence is often broadly defined as a property of a system that is not present in its microlevel parts, but that arises from their interaction (usually associated with serendipity, unexpected consequences, etc.). These emerging phenomena are also thought to form a "subset of the vast (and still expanding) universe of cooperative interactions that produce synergistic effects of various kinds" [3].

The purpose of this research is to initiate the examination of emerging institutions and service ecosystems in value cocreation processes under the basic tenets of S-D logic [5][6]. In this paper, we conduct several computational experiments, in which we represent the generic actors and their operant resources in an agent-based model, and examine their interactive behaviors in agent-based simulations. The agents do not have explicit, predefined roles at the beginning, such as fishermen, farmers, and marketers, but instead get identified with such roles and specific locations in their environment over time as a result of dynamic environmental conditions and evolving skills (influenced by opportunities and experiences) brought about by engaging in service-for-service exchange. The agents gradually form a cooperative society that is consistent with important features of S-D logic's service ecosystem.

\section{Related Work}

\subsection{S-D Logic}

Vargo and Lusch first introduced S-D logic in a paper in 2004 [14], in which they proposed an inverted 


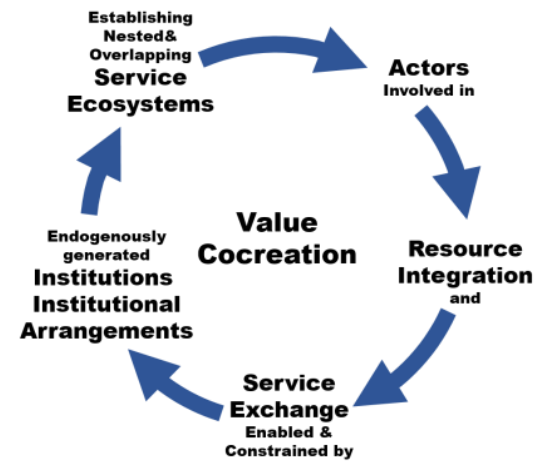

Figure 1. Core processes of service dominant

interpretation of economic activities traditionally explained from a goods-dominant and firm-centric viewpoint [10][16][19]. The basic concepts in S-D logic are interdependent; that is they are related to each other through recursive processes of value cocreation, as shown in Fig.1 [20]. Actors are fundamentally not different from each other, but vary in their levels of operant resources (e.g., skills and abilities). Service consists of the application of multiple resources (obtained from private, public and market sources) and are gathered and integrated by actors. Actors exchange service to satisfy their own requirements for living by helping others (thus increasing the viability of the system).

For service exchange to occur, actors depend on rules, called institutions. Some of these institutions are formalized (e.g. laws) and thus appear to be externally given, while others exist informally and endogenously emerge. All of them are, however selectively applied. These service activities, over time, stabilize value cocreating practices, resulting in discernible patterns. All emerge from actors' activities. In S-D logic, an interdependent structure among actors is called a service ecosystem, conceptualized in terms of reciprocally service-providing actors, coordinated by institutions. Value cocreation processes are recursive and change institutions and ecosystems dynamically. Such dynamism is embedded in value cocreation and service ecosystems -- relatively self-contained, selfadjusting systems of resource-integrating actors connected by shared institutional arrangements and mutual value creation through service exchange [18].

\subsection{Emergence and entropy}

There are various meanings and definitions of emergence in the scientific literatures. With respect to the question of causal discontinuity and whether or not emergence is compatible with reductionism, the terms weak and strong emergence have been applied [4]. Strong meanings of emergence generally denote the idea of causal discontinuity (from physical laws), and thus properties observed at the macro level, are intrinsically irreducible to and consequently unpredictable from (e.g., physical) properties at the micro level. Weak emergentists argue that emergent transitions are superficial at best. There are no new causal laws and the radical reorganization of parts are only due to their complex interactions. For example, Bedau discussed micro and macro level properties observed in emergence and provided a concept of weak emergence; "Weak emergence refers to the aggregate global behavior of certain systems. The system's global behavior derives just from the operation of micro level processes, but the micro-level interactions are interwoven in such a complicated network that the global behavior has no simple explanation" [1][2]. In emergence, the aggregation of micro level behavior generates discernible patterns of macro level phenomena. Stated differently, disorderedness at the micro level transpires into order observed at the macro level.

The identification of emergence is central to this paper, therefore we need a measure expressing how strongly emergence occurs. Entropy is a metric to represent disorder in a complex dynamic system [12][13]. There are several types of entropies proposed in various domains such as thermodynamic entropy, Boltzmann's entropy, social entropy, information entropy, generalized entropy, and so on. For example, in information entropy, $m$ indicates the number of an attribute's values that agents possibly have, and $p_{i}$ indicates the statistical probability of agents owning the $i$-th value of an attribute. We refer to the entropy $I$ in Eq. (1).

$$
I=-\sum_{i}^{m} p_{i} \log _{2} p_{i}
$$

Emergence $E$ can be defined as the decrease of entropy $I$ from the beginning of some process to the end:

$$
E=-\Delta I=I_{\text {start }}-I_{\text {end }}
$$

Gershenson defined a self-organization measure similar to Eq. (2) [7].

It is important that emergence restricts disordered states and reduces some kinds of entropies of the states. For example, actors are located so randomly at the beginning in a simulation where entropy is high, because the states are disordered. After several periods, actors limit their locations to small areas, and start generating groups with the same properties. At this stage, entropy decreases, because the actors' states become ordered and emerging patterns of actors arise 
in the simulation. As a result, the emergence of homogeneous groups (with respect to their roles) reduces entropy.

\section{Agent based simulation}

\subsection{Scenario}

In order to examine the explanatory power of S-D logic for emerging institutions and ecosystems in value cocreation, we keep the background scenario very simple as follows;

Actors reside on the land. In order to survive, they need certain amounts of both protein and carbohydrate. To obtain them, they need to catch fish and grow wheat, and sometimes cooperate with each other.

In this scenario, the value denotes to stay alive each period. In order to create value, each actor holds two basic types of skills, such as catching-fish and growing-wheat, which are represented as operant resources in S-D logic. Additionally, actors can exchange their resources and collaborate with each other and thus cocreate value. These interacting and integrating activities also require other types of operant resources, such as collaborating and exchanging skills. Collaboration is a skill to promote resource integration, while the skill to exchange promotes outcome resource integration.

\subsection{Agent modeling}

3.2.1. Base model. We modeled properties of an agent according to S-D logic as follows [6]:

- Actor:

- An actor is an autonomous agent in the simulation.

- Operand Resource:

- Fish is a resource that provides protein.

- Wheat is a resource that provides carbohydrates.

- Land is a locational resource.

- Operant Resource:

- Catching fish is a skill of an actor.

- Growing wheat is a skill of an actor.

- Exchanging is a skill of an actor.

- Collaborating is a skill of an actor.

- Fertilizing is the fertility level of land.

3.2.2. Skill and effort. Throughout the simulations, no particular role, such as fisherman or farmer, is formally assigned to an actor. We use the terms fisherman and farmer only to denote their relative levels of capabilities but not to impose in what activities they "should" engage. Initially, we only (randomly) assign capability levels and locations to actors. More specifically, a capability consists of two parts, i.e. skill and effort. The skill is an absolute level for doing a task. Each actor has a different skill level that allows it to achieve different performances in a task. As a result, it obtains different resultant outcomes corresponding to its skill level. The effort is a relative value that splits an actor's workload (at each iteration or period) into how much time it invests in obtaining one or the other resource (thus the ratio of the use of his operant resources). The total amount of effort for using the operant resources that an actor holds is summed to 1 . Following this restriction for the total effort, each effort value (for fishing, farming and exchanging) is initially assigned with a uniform distribution between 0 and 1, whereas the total amount of effort is canonicalized to 1.0. A particular capability (e.g. growing wheat) is calculated by multiplying an actor's skill value (e.g. to grow wheat) by the effort value to obtain the same resource.

3.2.3. Inheritance. All activities in the simulation are controlled by period and devolved through generations. In every period, an actor must ingest certain amounts of protein and carbohydrate to survive; otherwise it dies. Each actor also has a lifespan and at the end of the lifespan it dies. An actor periodically generates a child actor, whose properties are inherited from the parent actor with some fluctuations.

When inheriting the capability value, which consists of effort and skill values, and location from a parent, inherited values involve the following Gaussian noise in the later experiments:

- An effort value of a child actor $e^{\text {child }}$ follows the normal distribution $N\left(e^{\text {parent }}, 0.1^{2}\right)$.

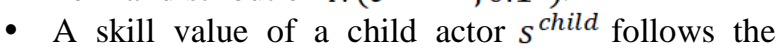
normal distribution $N\left(s^{\text {parent }}, 0.1^{2}\right)$.

- A location of a child actor $\operatorname{loc}_{x}^{\text {child } \text { or } y}$ follows the normal distribution $N\left(\operatorname{loc}_{x \text { or } y}^{\text {parent }}, 3^{2}\right)$.

The sum of effort values for the operant resources is canonicalized to 1.0 afterwards. The inheritance rules assign values to actors once, and then these values are unchanged through the actor's lifespan.

In each period, actors expend effort to obtain protein and carbohydrate to survive. Over time the map of the world gradually changes, partly due to actor deaths and births and partly because only good properties and locations are inherited by descendants. Individual actors' locations are not changed through the 
actors' lifespans; child actors reside close to the locations of their well-performing parent with the Gaussian distribution noise. Then, natural selection leads to a change in population distribution; it is an evolutionary mechanism.

3.2.4. Exchange. If an actor has capabilities high enough to obtain a necessary amount of fish and wheat, it can survive by itself, but if it does not have one or the other part, it has to find another actor for resource exchange.

- When an actor has a surplus amount of fish but has an insufficient amount of wheat, it needs to find an actor who has a surplus amount of wheat but has an insufficient amount of fish and exchange its outcomes to satisfy its respective conditions for survival.

- When an actor neither has sufficient amounts of fish nor wheat, it needs to play a role of a marketer, that is, a mediator to help other actors to exchange their resources. The marketer earns a part of the exchanging resources as a reward for mediation and cumulates amounts of resources to satisfy its condition for surviving.

The exchange skill is also an operant resource, measured as a capability. If an actor has a higher exchange capability, the actor can visit more actors who live far from the actor, and get larger amounts of rewards.

3.2.5. Collaboration. The process of catching fish holds several sub-tasks or sub-roles, such as preparing a fishing net, operating a fishing boat, finding schools of fish, and catching fish. Although an actor can do all these tasks by itself, it is sometimes inefficient. If several actors collaborate and share their tasks as a group, they can obtain outcomes more efficiently than the sum of stand-alone activities by themselves. Such activities require a collaboration skill among members of the group.

In order to explain the collaboration skill, we, at first, start representing a base level of an actor's operant resource for catching fish or growing wheat in the actor's effort $e_{i t}$ and skill $s_{i t}$, where $i$ is an index of actor and $t$ is a type of operant resources, such as fish or wheat. When a land fertility for type $t$ at location $(x, y)$ is represented as $f_{t}(x, y)$, then the actor's basic performance $p_{i t}(x, y)$ is expressed in Eq.(3).

$$
p_{i t}(x, y)=e_{i t} s_{i t} f_{t}(x, y)
$$

If this actor collaborates with an actor $j$, its performance becomes larger than its original

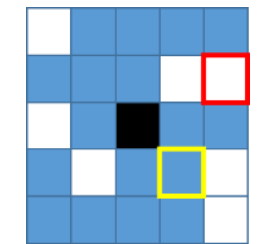

Figure 2. Collaboration partners

performance, but if the collaborator focuses on another type of work, such as fishing or farming, from the actor, the effect of collaboration gets smaller than that with the same-type collaborator. Additionally, an actor also has capability value for collaborating, denoted as $c_{i}$, where $i$ is an index of actor. In order to satisfy all constraints described here, we define the performance with collaboration by calculating the original performance multiplied by $\left(1+c_{i} c_{j} e_{j t} s_{j t}\right)$.

$$
p_{i t}(x, y)=e_{i t} s_{i t} f_{t}(x, y)\left(1+c_{i} c_{j} e_{j t} s_{j t}\right)
$$

Moreover, if this actor collaborates with two actors $j$ and $k$, its performance should be multiplied by $\left(1+c_{i} c_{j} e_{j t} s_{j t} / 2\right)\left(1+c_{i} c_{k} e_{k t} s_{k t} / 2\right)$. Generally stated, the actor's performance is calculated as Eq.(5), when $m$ is the number of collaborators.

$p_{i t}(x, y)=e_{i t} s_{i t} f_{t}(x, y) \prod_{j=1}^{m}\left(1+\frac{c_{i} c_{j} e_{j t} s_{j t}}{m}\right)$

Final outcomes in a simulation period involves Gaussian noise, and are calculated in $p_{i t}$ multiplied by a fluctuation function given as Gaussian distribution.

Locations of collaborators are limited to a small square around the focal actor. Fig. 2 shows a sample map around an actor in a square whose width is limited to 5. The black square located at the center is location where the focal actor resides. A blue square indicates that an actor resides on it, and a white square indicates that no actor resides on it. As an example, two squares shown as red and yellow edges are selected as collaborators. There is an actor at yellow-edged square, but no actor at red-edged square, then collaboration is successfully done only with the actor on the yellow square. When the actor on the yellow square has $e_{j t}=0.8, s_{j t}=0.5, c_{j}=1.0$, and $c_{i}=1.0$, then the performance is multiplied by $1+1.0 \times 1.0 \times 0.8 \times 0.5 / 2=1.2$.

Although an actor selects $m$ collaborators within a diameter from its residential location, basically at random, the simulator optionally memorizes the partners collaborated with in the past. If the currently selected partner has worse performance than the partner in the past, and the memorizing option is set as 
true, then the past partner is finally selected as a collaboration partner. Additionally, a memorized partner navigates to find better partners by introducing the partner's partners. This navigation makes collaboration networks larger than the original collaboration diameter.

The more actors reside around the focal actor and hold the same operant resources, such as catching-fish or growing-wheat, the higher expected effect (gains) of the collaboration for the focal actor arises. If few actors reside around the focal actor or the actors' preferable operant resources are different from the focal actor, the expected effect of the collaboration becomes smaller. Therefore, the residential location and surrounding partners' preferences are very important for collaboration.

\subsection{Land fertility}

A world map is given as a cell structure, and each cell involves zero or one actor. The shape of the world map is a torus, where the right edge is connected to the left edge, and the top edge is connected to the bottom edge. The map size is $200 \times 200$. An actor who obtains 1.0 fish and 1.0 wheat in a period can survive on the land and generates a child actor every five periods.

If the land is very fertile, actors can survive by themselves, and no resources are integrated among them. Since we would like to observe an evolutionary selection as a result of resource integration, the fertility level of the land is inevitably set at a poor level. If the fertility level is poor, most actors whose properties are randomly assigned immediately die due to lack in their outcomes. To avoid immediate death, we necessary set a fertile area at the center of the land where initial actors start living, and set a poor condition at the outer area. The radius of the area is 10 units, and the initial number of actors is set to 50 .

Fig. 3 shows a sample of snapshots of the history of the simulation map, where each cell color is determined as the actor's efforts for the activities. Fishing, farming and exchanging efforts are expressed in brightness of blue, red and green. In the early stage, actors live together within the center circle, and then start expanding their residential areas outside the circle. During the expansion, actors transit toward fishermen (blue) or farmers (red). Residential areas are expanding in a large circle. In Fig.3(c), marketers (green) come out at the left hand side, and help other actors to exchange their outcomes.

The map is not always explicitly divided into fishermen, farmers and marketers. It depends on the environments, such as fertility levels of the land. In Fig.4, different fertility levels expressed in ' $f$ '. generate different distributions of roles. ' $f=2.0$ ' is a fertility

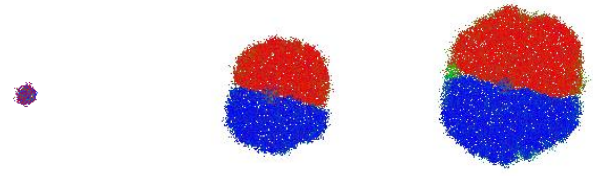

(a) Initial stage (b) Middle stage (c) Final stage Figure 3. Sample of growing population

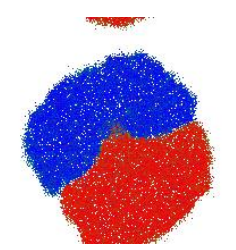

(a) $f=1.4$

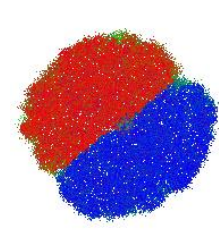

(b) $f=1.6$

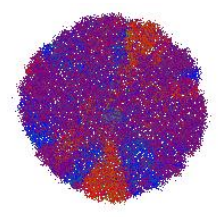

(c) $f=1.8$

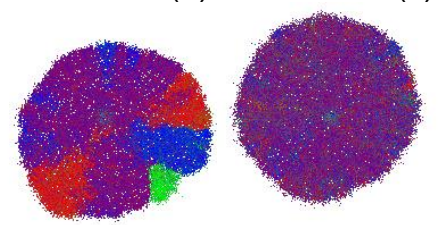

(d) $\mathrm{f}=2.0$

(e) $f=3.0$

Figure 4. Different fertility levels

level where the actor obtains 2.0 outcome in a period without collaboration if an actor's skills for fishing and farming are both 1.0. If an actor obtains 2.0 fish, it can exchange 1.0 fish to 1.0 wheat, and stay alive. If a fertility level is less than 2.0, actors need to collaborate with other actors. In a case of $f=1.4$ or 1.6 in Fig.4, areas of fishermen and farmers are clearly separated. It indicates that they need to strongly specialize and collaborate their tasks to work efficiently and then exchange their outcomes with the opposite typed actors. On the other hand, in the case of $f=2.0$ or 3.0 , most cells are filled with dark purple color that consists of a half blue and red. It indicates that the actors do not specialize their tasks and work for both fishing and farming by themselves. Because of the richness of the land, the actors keep their lives by themselves without specialization and exchange.

\subsection{Calculation model for entropy}

We propose two types of calculation models for entropy; spatial and relational entropies. First, we define an actor's type as a type of operant resource, for which the actor spends most effort. Spatial entropy is based on randomness of adjacent neighbors' types. For the focal actor and eight-neighbor actors, it counts the number of actors for each type, calculates shares for each type, and then calculates entropy by Eq. (1). Relational entropy is based on partner relationships. It is similar to spatial entropy, but calculates it for the focal actor and its collaboration partners. The current 
simulator deals with three actor types, such as fisherman, farmer and marketer. If the three types evenly exist in a group, the entropy is 1.58 . If all actors are the same type, the entropy is 0 .

\section{Institutions}

Actors in a society work with collaborators and exchange their resources with other actors. Such social actions are constrained with locally governed rules and customs; that is institutions. In the simulation, local groups, such as fishermen or farmer groups, emerged as a result of aggregating micro behaviors of actors. These emergent groups point towards endogenous institutions. For example, an actor living in the fishermen group should work better as a fisherman, because the actor has more chances to collaborate with neighbors. On the other hand, if the actor behaves as a farmer, the actor must work alone without help, thus the outcomes become smaller. The actor's specialty is not only dependent on its talent but also on its surrounding environments. The institutions are not guaranteed as optimum solutions, but reasonably fitting to the surrounding situations.

In S-D logic, institutions are endogenously generated. There are two types of ways to generate institutions.

- Micro to macro generation: Aggregation of actors' micro behaviors spontaneously leads to a macro structure.

- Macro to micro generation: Actors' local recognition of surrounding environments enhances the macro structure of the institutions that constrain the actors' micro behaviors.

In the preliminary experiments, we found that specialized groups emerged that aid survival. This is a sort of emergent pattern from micro behaviors. If an actor has cognitive ability to recognize that groups increase chances of survival, actors might seek out groups in order to survive. Although actors hardly capture whole phenomena from a truly macro viewpoint, they can capture local patterns, and change their behavior toward more conducive conditions for survival. Following the above idea, we define two macro to micro institutions and examine their effects.

Rule 1: An actor counts up the number of neighbor actors (max. 8-neighbors). Then, the actor randomly selects a destination location in a limited distance, and counts up the number of neighbor actors in in the same way. If the destination has more actors surrounding, the focal actor moves to the destination.

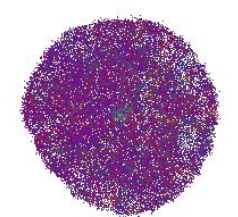

(a) Original

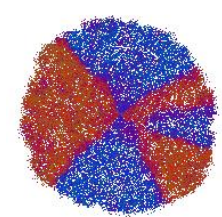

(c) Rule2

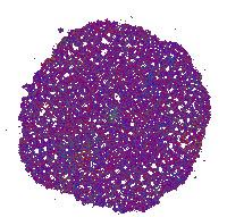

(b) Rule1

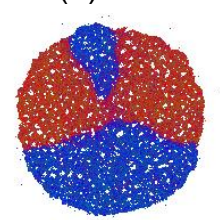

(d) Rules 1 \& 2
Figure 5. Different fertility levels

Rule 2: An actor categorizes 8-neighbors with their specialties, such as fishermen, farmers, and marketers. If more than half belong to the same category, the actor adapts its effort level to the majority. For example, if the actor finds five fishermen in 8-neighbors, it raises its fishing effort even if its farming skill is very high.

In these rules, an actor does not directly recognize a group, but the actor tends to move toward a location where more actors reside, or to change its working effort to make its own resource integrating with neighbors' resources smoothly. As a result, actors or descendants of the actors tend to aggregate together to keep their lives longer.

Fig.5 shows results of applying the macro institutions to actors. The fertility level is set so high, such as 3.0, that the original model does not generate groups. However, when rule 2 is applied, shown in Fig.5(c), groups appear. If both rules 1 and 2 are applied, the boundary of groups becomes clearer, shown in Fig.5(d).

\section{Experiments and analysis}

\subsection{Transitions in social structures}

5.1.1. Early stages. At the initial state, 50 actors are located in a small circle at the center of the map. Within the circle, the land is so fertile that actors can easily keep their lives as they obtain a sufficient amount of fish and wheat. They are relatively independent from others and rarely exchange their services. During some thousands periods, depending on the given conditions, actors remain in the circle, and are gradually changing their internal properties, such as skill levels and their collaboration partners, but it remains hard to migrate from the circle, because the land out of the circle is too severe to live. However, the circle is so small that its growth inevitably stops. 
Figure 6. Snapshot of actors' spouting out

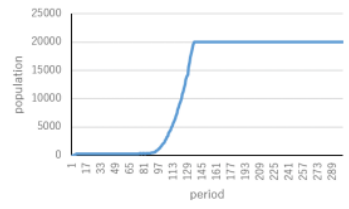

(a) Population

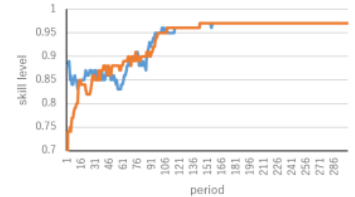

(c) Skill levels (b) Outcome

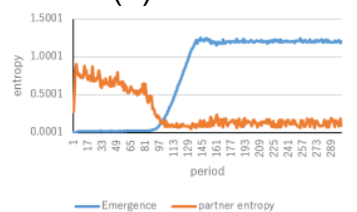

(d) Entropy

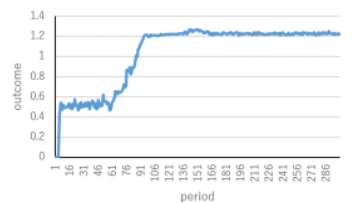

Figure 7. Sample of growing process

5.1.2. Transition stages. Even in the early stages, quite a few pioneers try to migrate, but most of them immediately die because of lack of resources. This fragile situation is broken down suddenly, perhaps, by an incidental co-occurrence of actors who are specialized into the identical skill and try to go outside. Fig.6 is a snapshot where a group of actors specialized in fishing(blue) are pouring out from the small circle. They can survive because they are tightly coupled with each others in collaborative resource integrating links. Additionally, another group specialized in farming(red) simultaneously appear at the different side of the circle as service exchange partners.

Such a phase transition process can be explained as dynamics of ecological systems described by Holling[9]. He proposed four phases in an adaptive cycle of ecosystems; phases of growth(r), conservation $(\mathrm{K})$, release of bound-up resources $(\Omega)$, and reorganization $(\alpha)$. The observed stages in Fig.6 are in a transition from conservation $(\mathrm{K})$ to release $(\Omega)$, and continue to a phase of reorganization $(\alpha)$.

Fig.7 shows a sample case of this phase transition process. The $\mathrm{X}$ axis is a period divided by 10 . Population started increasing after around 700 periods, shown as 70 in Fig.7. The population exponentially increased, and stopped at 20,000 that is the maximum number of the actors in a setting of a half of the map size, shown in Fig.7(a). The other graphs show changes of the actor's other properties. Fig.7(b) shows the change of average outcome levels of the actors who reside outside of the center circle. Before the phase

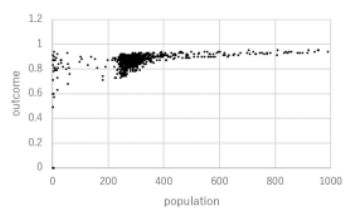

(a) Outcome

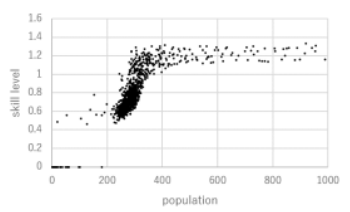

(b) Skill levels

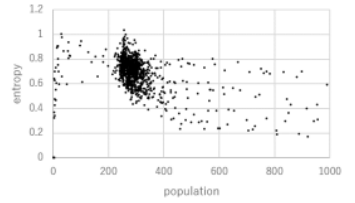

(c) Entropy

Figure 8. Canonicalized growing process

transition, the average outcomes are less than 1.0, so that most of actors cannot survive, but at the transition point, the outcomes are drastically improved, and then converged on 1.0. This shift corresponds to phases from release $(\Omega)$ to reorganization $(\alpha)$. Similar tendency is also observed in skill levels, shown in Fig.7(c). The skill levels gradually increase in a conservation phase $(\mathrm{K})$, and at the release phase $(\Omega)$, they reach the maximum skill of 1.0.

5.1.3. Growing stages. Fig.7(d) provides a different viewpoint in terms of entropy and emergence. In Fig.7(d), entropy is the relational entropy calculated by counting types of collaboration partners. Before the phase transition, all actors resided in the circle and had various types of collaborators around them, but after the transition, their neighbors became almost identical so that the entropy converged into zero through the growth(r) and conservation(K) phases. Emergence is calculated by the differences of the spatial entropy between the observed state and the completely disordered state calculated in the theoretically-random model. Emergence levels increased during the conservation $(\mathrm{K})$, because actors were specialized and aggregated into the same types.

5.1.4. General behaviors. Fig. 8 shows the general process of the phase transition. In these graphs, $\mathrm{x}$-axes are canonicalized to the size of the population. Because the radius of the center circle is 10 , the size of cells within the circle is around 314. Therefore, the transition are always observed at the stages from 300 to 500 actors. In Fig.8, the number of actors and the indicators were plotted as results of ten simulations conducted. These graphs show similar tendencies observed in a sample process shown in Fig.7, such as raising outcomes and skill levels, and decreasing entropy levels.

\subsection{Micro to macro emergence of institutions}




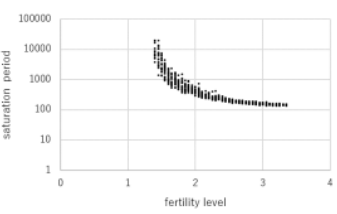

(a) Growing Periods

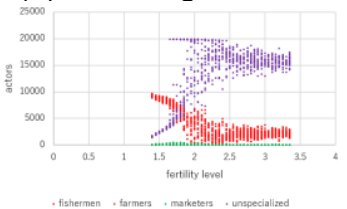

(c) Population for types

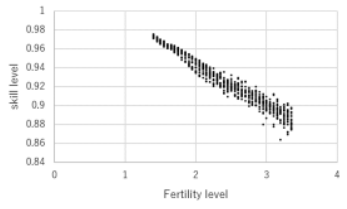

(b) Skill levels

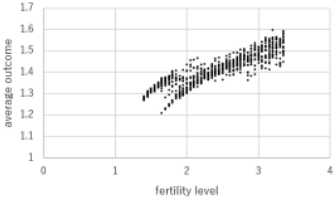

(d) Average Outcome

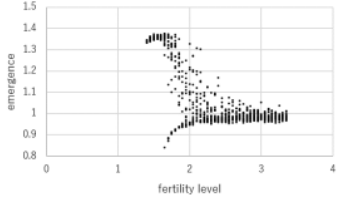

(e) Entropy

Figure 9. Canonicalized growing process

Institutional emergence is partially a function of environment, such as fertility of the land. This subsection examines in more depth the effect of fertility levels from 1.4 to 3.4 with 0.05 pitch. For each fertility level, we conducted 20 simulations; Totally 800 simulations were conducted. We observed actors' properties when the number of actor reached 20,000.

5.2.1. Growth rate and skills. Fig.9(a) shows the periods taken to reach the end. As fertility levels were raised, the periods became shorter because fertile lands provide environments suitable for increasing population. Fig.9(b) shows the skill levels obtained by the specialized actors. As the fertility levels were raised, the skill levels decreased. This is because fertile lands do not require higher skill levels for obtaining a sufficient amount of resources.

5.2.2. Path dependencies. Fig.9(c) shows the population for each specialization. In this graph, fishermen and farmers are drawn in the same color: red, because these two specialized actors have no difference in observation. Green marks represent marketers, and purple marks express actors that are not specialized. It is remarkable that two separate modes come out in lower fertility levels. Purple marks are divided into almost 20,000 and the others. Red marks are also divided into 5,000-10,000 levels and almost zero, which is hard to see in the graph, because green marks are overdrawn. In other words, specialized actors are dominant and unspecialized actors are rare, in one mode, and specialized actors are rare and unspecialized

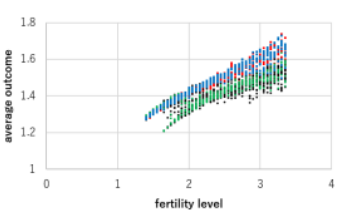

(a) Outcome

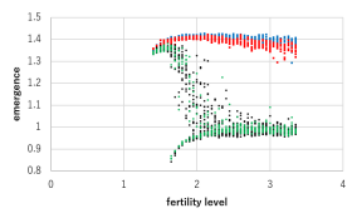

(b) Entropy
Figure 10. Institutions by local recognition
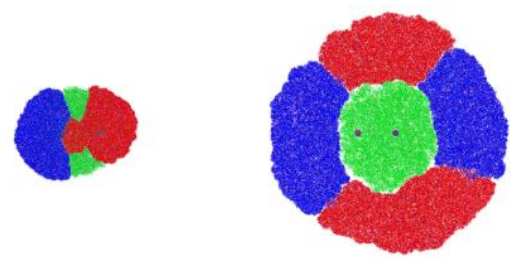

$\begin{array}{ll}\text { (a) Middle stage } & \text { (b) Final stage }\end{array}$

Figure 11. Large scale simulation

actors are dominant, in another mode. These modes non-deterministically appeared by the random seeds. When the phase transition starts with two distinct specialized groups of actors, these specialized actors become dominant. When it starts with unspecialized actors, the unspecialized actors become dominant. These two modes are also observed in average outcome and emergence graphs shown in Fig.9(d) and (e).

\subsection{Macro to micro emergence of institutions}

As described in Section 4, local pattern recognition enables actors to generate institutions in manners of macro-to-micro effects. Fig.10(a) shows average outcomes and Fig.10(b) shows emergence. The original model is represented with black colored points, the rule 1 is with green, the rule 2 is with red, and the combination of rules 1 and 2 is with blue. The rule 2 and the combination are marked at higher cluster in the graphs of Fig.10(a) and (b). This indicates that rule 2 and the combination generate highly separate groups with specialization as an institution, and obtain higher outcomes. It suggests that local pattern recognition enables actors to generate stronger institutions.

\subsection{Large scale simulation}

It used 800 x 800 cell map. As an initial state, we set two fertile circles located close to the center. Fig.11 shows the maps of middle and final stages after the long run. At the middle stage of the first conservation phase $(\mathrm{K})$, specialized marketers came out at the edge of the living area as a result of necessity for many actors to rely on the marketers for their service exchange, because the market became larger. This led 
to the second level reorganization phase $(\alpha)$ where the marketers dominate service exchange markets. The marketers gradually moved to the center of the map because the marketers must interact with many fishermen and farmers to exchange resources. This process corresponds the second conservation phase $(\mathrm{K})$.

There was another case with different parameters, where most actors suddenly died after the growth. The residential area shrunk to the original center circle and started growing again. It seemed that a balance of mutual dependencies among actors was partially collapsed at first, and it was spread over the whole, like the collapse of the bubble economy.

\section{Discussion}

\subsection{Institutions and ecosystems in S-D logic}

This section discusses observed phenomena in the experiments along the concepts described in Fig.1.

6.1.1. Actor and specialization. No special roles are assigned to any actor at the initial stage, but actors are gradually specializing by a process of evolutionary selection. The current simulator does not permit actors to change their efforts and skill levels for operant resources in their lifespan except in the case of local pattern recognition experiments, but an evolutionary selection mechanism selects specialized actors as survivors.

6.1.2. Resource integration and service exchange. There are two types of resource integration, i.e. exchange and collaboration. In exchange, actors' outcomes are exchanged for complementing their obtained operand resources. In collaboration, actors' skills are specialized in a part of activities, and shared among collaborative actors. The outcomes and skills are also defined as operand and operant resources in $\mathrm{S}$ $\mathrm{D}$ logic, thus these exchanging and collaborative activities are both recognized as service exchange.

6.1.3. Institutions. At the beginning, the simulation field is flat and no distinction exists on the field. While actors are selected via an evolutionary mechanism, special groups, such as fisherman and farmer groups, appear. In a fisherman group, an actor behaves better as fisherman, because there are more opportunities to obtain more outcome to stay alive. This is a sort of local institution. Institutions are dynamically configured through evolutionary process of the simulation, and dynamically changed in their environments.

An institution is a macro-level observation generated from micro-level activities. On the other
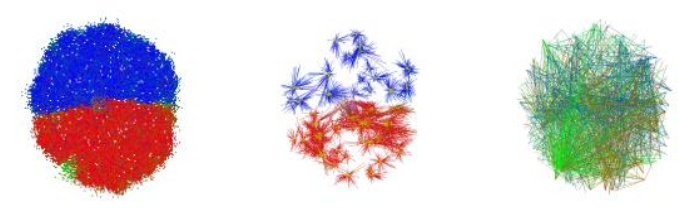

\section{(a) population (b) Collaboration (c) Exchange Figure 12. Service ecosystems}

hand, if a macro-level institution from local viewpoint is recognized by the actors and a rule is applied for the actors to enhance the institution, micro-level behaviors of the actors might change. In the experiments, when the macro institutions which enforce actors to come close to each other was assigned or change their roles to adjust to the neighbors, groups were intensively generated. As shown in this result, micro behaviors and macro institutions are correlated with each other and configure social systems, called service ecosystems.

6.1.4. Service ecosystems. We observed dynamics of service ecosystems in forms of phase transitions. As a background of the transitions, there are social networks where actors gather around strong actors and generate huge groups. Fig.12 consists of three drawings. The left hand is a population map, the center is a collaboration link map, and the right hand is an exchange link map. It shows that collaboration network inside the groups and exchange network between the groups are complicatedly mixed, and configure service ecosystems.

Exchange and collaboration are both important to make the service ecosystems stable. Collaboration is a process integration of resources, and it corresponds to a structure of organizations in the real world. On the other hand, exchange is an outcome integration of resources, and the exchange network is relatively dynamic in terms of its configuration. Both are tightly coupled with each other, and keep their total structures stable.

\subsection{Emergence}

Helbing explains the emergence of cooperative or coordinating norms in a game theoretic situations with agent preferences [8]. His methodology is similar to our research, because the world is a grid, and agents work in time series of the world. However, agents are cognitive in a sense of determining their behaviors against their profits in his model. Additionally, his model does not have complex social networks, like exchanging and collaborating networks.

Emergence has been formalized in many ways described in Section2.2. We followed the definition of weak emergence proposed by Bedau [1][2], and 
defined the emergence in terms of entropy in Eq.(2), referring to Gershenson's work[7]. This formalization worked well in an S-D logic simulation especially for analyzing dynamism in service ecosystems and institutionalization.

\section{Conclusion}

This paper examined a quantitative explanation of S-D logic using agent based simulation. We modeled an actor as an agent, and defined operant resources in it. The operant resources were measured in skill and effort values. At the beginning, the skill and effort values were randomly assigned to actors, but they were gradually modified through evolutionary selection, and finally were specialized into specific roles. We also introduced two types of resource integration skills, such as exchange and collaboration, which contributed to create institutions and service ecosystems. Sample scenarios were very simple, but we succeeded to explain some parts of phenomena expected in S-D logic, such as emerging endogenous institutions and the formation of service ecosystems. The actors mutually interacted with each other and successfully cocreated value. The proposed models and scenarios were so simple that we should expand them in future and analyze in greater detail.

\section{References}

[1] M. Bedau, "Weak emergence," Philosophical Perspectives: Mind, Causation, and World, 11:375--399, 1997.

[2] M. Bedau, "Downward causation and the autonomy of weak emergence," Principia, 6(1) 5--50, 2002.

[3] P. A. Corning, P. A. The re-emergence of emergence: A venerable concept in search of a theory. Complexity, 7, 18-30, 2002 .

[4] T. W. Deacon, "Incomplete Nature: How Mind Emerged from Matter"; W. W. Norton Company: New York, NY, USA, 2011,

[5] S. Fujita and Y. Kase, "Service market simulation based on service dominant logic," IEEE International Conference on Agents, pp. 31-36, 2016.

[6] S. Fujita, C. Vaughan and S. L. Vargo, "Service ecosystem emergence from primitive actors in service dominant logic: An exploratory simulation study," Hawaii International Conference on System Sciences, pp. 1601-1610, 2018.
[7] C. Gershenson and N. Fernández, "Complexity and information: Measuring emergence, self-organization, and homeostasis at multiple scales," Complexity, 18(2):29--44, 2012.

[8] D. Helbing, W. Yu and K-D. Opp and H. Rauhut, "Conditions for the Emergence of Shared Norms in Populations with Incompatible Preferences," PLoS One, 9(8), 2014.

[9] C. S. Holling, "Understanding the Complexity of Economic, Ecological, and Social Systems", Ecosystems, Vol.4, pp. 390-405, 2001.

[10] R. F. Lusch and S. L. Vargo, "The Service-dominant Logic of Marketing: Dialog, Debate, and Directions". Sharpe, 2006.

[11] R. F. Lusch and S. L. Vargo, "Service-Dominant Logic: Premises, Perspectives, Possibilities," Cambridge University Press, 2014.

[12] M. Mnif and C. Muller-Schloer, "Quantitative Emergence,” pp. 39--52. Springer Basel, 2011.

[13] M. Tang and X. Mao, "Information entropy-based metrics for measuring emergences in artificial societies," Entropy, 16(8):4583-4602, 2014.

[14] S. L. Vargo and R. F. Lusch, "Evolving to a new dominant logic for marketing," Journal of Marketing, vol. 68, no. 1, pp. 2-17, 2004.

[15] S.L. Vargo, and R.F. Lusch, "From goods to service(s): Divergences and convergences of logics." Industrial Marketing Management, 37(3), 254-259, 2008.

[16] S. L. Vargo and R. F. Lusch, "Service-dominant logic: Continuing the evolution," Journal of the Academy of Marketing Science, no. 36, pp. 1-10, 2008.

[17] S. L. Vargo. and R. F. Lusch. "It's all B2B and Beyond . . . : Toward a Systems Perspective of the Market," Industrial Marketing Management, 40 (2) 181-187, 2011

[18] S. L. Vargo, H. Wieland and M. A. Akaka, "Innovation through institutionalization: A service ecosystems perspective," Industrial Marketing Management, vol. 44, pp. 63-72, 2015.

[19] S. L. Vargo and R. F. Lusch, "Institutions and axioms: an extension and update of service-dominant logic," Journal of the Academy of Marketing Science, vol. 44, no. 1, pp. 523, 2016 .

[20] S. L. Vargo and R. F. Lusch, "Service-dominant logic in 2025," International Journal of Research in Marketing, vol. 34, no. 1, pp. 46-67, 2017. 